\title{
A implantação em larga escala da Estratégia de Saúde da Família na cidade do Rio de Janeiro, Brasil: evidências e desafios
}

\author{
Large-scale implementation of the Family Health Strategy \\ in the city of Rio de Janeiro, Brazil: evidence and challenges
}

Nilson do Rosário Costa (https://orcid.org/0000-0002-8360-4832) ${ }^{1}$
Iandara de Moura Silva (https://orcid.org/0000-0002-2391-0977) ${ }^{1}$
Paula Travassos de Lima (https://orcid.org/0000-0001-8505-6801) ${ }^{2}$
Thaís Severino da Silva (https://orcid.org/0000-0002-8055-9992) ${ }^{2}$
Isabel Cristina Maria da Costa (https://orcid.org/0000-0002-9188-7748) ${ }^{3}$
Iara Veloso Oliveira Figueiredo (https://orcid.org/0000-0002-0927-859X) ${ }^{4}$

${ }^{1}$ Departamento de Ciências Sociais, Escola Nacional de Saúde Pública, Fundação Oswaldo Cruz. R. Leopoldo Bulhões 1480, Manguinhos. 21041-210 Rio de Janeiro RJ Brasil.

nilsondorosario@gmail.com

${ }^{2}$ Secretaria de Estado de Saúde do Rio de Janeiro. Rio de Janeiro RJ Brasil.

${ }^{3}$ Fundação de apoio à

Fiocruz (Fiotec). Rio de Janeiro RJ Brasil.

${ }^{4}$ Instituto René Rachou

Fiocruz Minas. Belo

Horizonte MG Brasil.

\begin{abstract}
The city of Rio de Janeiro has implemented, on a large scale, the model of Social Organizations (OSS) for the management of Primary Health Care (PHC). This option makes the understanding of the city's experience very relevant, especially since, until then, the OSS organizational model had been adopted predominantly in the SUS hospital management. Thus, the experience of PHC development at two conflicting moments of municipal management in relation to the OSS model is analyzed: the implementation and development of the PPPs (2009-2016); and their dismantling (2017-2020). Case Studies, Literature Review and analysis of public data from DATASUS/Ministry of Health were used. It was verified that: the adoption of outsourcing based on OSS can be directly associated with the rapid expansion of PHC in the city and PHC coverage improvement indicators; the diffusion of the OSS model is associated with the high priority given to health expenditures in the municipal budget; the sustainability of the adoption of the OSS model did not depend on the municipality's economic status, but on the government's political choice in the period of 2009 to 2016. The PPP arrangement resulted in important organizational advances, although it did not prevent the veto of the OSS model carried out during the 2017-2020 term.
\end{abstract}

Key words Family Health Strategy, Primary Care, Financing, Budgetary Binding, Large Cities
Resumo A cidade do Rio de Janeiro implantou em larga o modelo das Organizações Sociais (OSS) para a gestão da Atenção Primária à Saúde (APS). Esta opção torna a compreensão da experiência da cidade muito relevante, especialmente porque até então o modelo organizacional das OSS tinha sido adotado dominantemente na gestão hospitalar do SUS. Assim, é analisada a experiência de desenvolvimento da APS em dois momentos conflitantes da gestão municipal em relação ao modelo das OSS: a implantação e desenvolvimento das PPP (2009-2016); e o seu desmantelamento (2017-2020). Utilizou-se o Estudo de Caso, Revisão Bibliográfica e análise dos dados públicos do DATASUS/Ministério da Saúde. Identificou-se que: a adoção da terceirização com base nas OSS pode ser diretamente associada à rápida expansão da APS na cidade e a melhoria de indicadores de cobertura da APS; que a difusão do modelo das OSS está associada à alta prioridade dada ao gasto com saúde no orçamento municipal; que a sustentabilidade da adoção do modelo da OSS não foi dependente da condição econômica do município, mas da escolha política do governo no período 2009-2016. O arranjo das PPP produziu avanços organizacionais importantes, mas não impediram o veto ao modelo das OSS levada adiante ao longo da gestão de 2017-2020.

Palavras-chave Estratégia de Saúde da Família, Atenção Primária, Financiamento, Vinculação Orçamentária, Grandes Cidades 


\section{Introdução}

O presente artigo tem por objetivo analisar a implantação em larga escala da Estratégia de Saúde da Família (ESF) no âmbito do Sistema Único de Saúde (SUS) na cidade do Rio de Janeiro nas duas últimas décadas. A utilização de serviços de saúde no Brasil pela população pobre permanece diretamente associada a provisão do SUS, como argumentaram Faveret Filho e Oliveira ${ }^{1}$. A relevância do estudo do caso do Rio de Janeiro devese à expressiva expansão da provisão de serviços públicos e gratuitos durante os dois mandatos do Prefeito Eduardo Paes (2009-2016) ${ }^{2}$, que contrariou as expectativas da literatura sobre a impossibilidade de política expansionista para a atenção primária (APS) nas grandes cidades brasileiras.

Em 2010, a cidade do Rio de Janeiro tinha 6.320.446 habitantes, sendo o segundo município mais populoso do país ${ }^{3}$. O déficit extremo na oferta de serviços de saúde caracterizava os territórios onde vive a população pobre ou de baixa renda da cidade. A ampliação da oferta de serviços públicos de atenção básica no SUS tem repercussão positiva no bem-estar coletivo, como reconhece a literatura brasileira que compreendeu a natureza redistributiva da política da atenção básica ${ }^{4}$.

Cabe chamar a atenção que a cidade do Rio de Janeiro se destacou nas décadas de 1990/2000 pela baixa capacidade de ampliação dos serviços de atenção primária, com estagnação da oferta e do financiamento público ${ }^{5}$. Esperava-se que a expansão da APS na década de 1990 pudesse mitigar a situação de desigualdade. Entretanto, constatou-se que a meta da ampliação da provisão de serviços e ações da APS foi alcançada nas pequenas e médias cidades, com destaque para os municípios com baixo Índice de Desenvolvimento Humano. Entretanto, os municípios brasileiros de grande porte aderiram residualmente à ESF. A insignificante implantação nas grandes cidades foi considerada como uma limitação da estratégia de ampliação da afetividade do SUS. O Rio de Janeiro era um exemplo expressivo porque em 2008 apenas 3,5\% da população estava coberta pela $\mathrm{ESF}^{6}$. Esta condição da cobertura nas grandes cidades era crítica em razão da ESF ser considerada a porta preferencial de acesso aos serviços gratuitos do SUS.

As grandes cidades tornaram-se, naquela conjuntura, alvo de uma política específica de incentivo: o Projeto de Expansão e Consolidação da Saúde da Família (PROESF), implantado em 2003. O PROESF explicitava, portanto, o descon- forto associado ao fato de que importantes cidades de grande porte populacional apresentassem cobertura de Saúde da Família insignificante em fins da década de 2000. A baixa cobertura da ESF suscitou muitas interrogações sobre as condições inibitórias da adesão das grandes cidades ao modelo proposto pela ESF5.

Parcela da literatura concluiu que os incentivos federais anteriores a Emenda Constitucional 29 (EC-29) de 2000, que vinculou as despesas públicas com ações e serviços públicos de saúde (ASPS) ${ }^{7}$, foram insuficientes e que a necessidade de contrapartida financeira municipal para o custeio inibiu a expansão da ESF nos grandes Municípios. Assim, a escala da cobertura da ESF dependeu exclusivamente da capacidade econômica do Município. Na federação brasileira, considerou-se que a ESF geraria incentivos iguais a todos os Municípios para aderir às preferências do governo central. A indução do governo federal e dos estados à implantação de grande número de equipes transferia o ônus financeiro principal ao Município. As transferências não seriam, portanto, suficientes para pagar os custos totais das equipes. Os incentivos lineares utilizados pelo Ministério da Saúde não seriam adequados aos Municípios, diferenciados financeiramente ${ }^{5}$.

A segunda corrente explicativa considerou que cidades já com elevado estresse das despesas com ações e serviços de saúde na execução financeira tenderiam a limitar os gastos com a expansão da ESF por força das restrições legais que impediriam o crescimento das despesas do governo municipal especial como pessoal. A expansão da ESF submeteria o gestor público ao risco de descumprimento da Lei de Responsabilidade Fiscal (Lei Complementar $n^{\circ}$ 101/2000) para o gasto com pessoal por força da exigência da época de que as equipes da ESF fossem compostas por servidores públicos estatutários em regime de contrato de trabalho de 40 horas semanais ${ }^{5}$.

Nesse contexto, este artigo demonstra e reflete sobre as escolhas realizadas pela cidade do Rio de Janeiro para contornar as restrições e os dilemas identificados na literatura para a expansão em larga escalas da ESF, que exige a função saúde no centro da agenda pública da cidade.

É importante frisar que a implantação em larga escala de uma política pública no SUS pode apresentar enorme instabilidade em razão da possibilidade de veto de coalizões em posição de competição política com os implementadores. Como chama atenção Tsebelis ${ }^{8}$, a instabilidade de uma política é diretamente proporcional a discrepância de valores ideológicos entre compe- 
tidores. Não há dúvida que a disputa programática faz a diferenças na competição eleitoral no Brasil ${ }^{9}$.

As políticas no âmbito SUS podem, assim, apresentar elevado grau de volatilidade institucional em razão do conteúdo da inovação proposta pelo governante. Uma característica importante da gestão Paes na saúde foi a adoção do modelo de terceirização com base nas Organizações Sociais (OSS) para a expansão da ESF, objeto de veto de competidores políticos, comunidade epistêmica dos sanitaristas e servidores estatutários. Ao fim de dois mandatos (2009-2016), Eduardo Paes não teve sucesso em fazer o sucessor no governo da cidade. Ascendeu a coalizão políticas em torno do Prefeito Crivella (2017-2020), que implementou o veto às terceirizações por meio das OSS, embora seu programa de governo nunca tenha explicitado esta intenção ${ }^{10}$. A ação de veto é definida pela introdução de nova legislação ou arranjo administrativo que desestabiliza o curso da política pública vigente ${ }^{8}$.

Como exemplo de veto ao modelo, em 2018 a gestão Crivella publicou o documento "Reorganização dos Serviços de APS - Estudo para Otimização de Recursos”. Entre as medidas propostas, está a extinção de 184 ESF e 55 equipes de Saúde Bucal, com adesão de nova tipologia de equipes para $\mathrm{APS}^{11}$.

O documento se apoiou na justificativa de déficit no orçamento municipal, que culminou com a redução de cerca de 1.400 postos de trabalho na APS do município e iniciou a mudança do modelo de gestão por OSS, transferindo parte dos serviços e vínculos trabalhistas para a Empresa Pública - Rio Saúde em 202011.

No mesmo documento foi mencionada a produção das equipes de saúde da família, que em média cobririam um território inferior a 3.000 pessoas e, portanto, existiam profissionais ociosos. A avaliação desconsiderou a diversidade de conformações territoriais que a cidade apresenta, como, por exemplo, as áreas de sob o domínio do tráfico de drogas que impede a mobilidade das pessoas. Cabe considerar que neste período houve a mudança dos sistemas de informação que traziam os dados de cadastro dos usuários ${ }^{11}$.

Chamou a atenção também os critérios para redução de equipes em áreas consideradas privilegiadas (de classe média), desconsiderando a presença de comunidades pobres e favelas em tais bairros e a perda de plano de saúde.

\section{Materiais e métodos}

O estudo de caso focaliza duas dimensões importantes da experiência da cidade: 1) o uso dos mecanismos de terceirização para o desenvolvimento acelerado da atenção primária, e 2) e a vinculação do orçamento da cidade para assegurar sustentabilidade do financiamento ASPS. O estudo de caso enfatiza três momentos da gestão pública municipal: o primeiro e o segundo mandatos do Prefeito Eduardo Paes, respectivamente 2009-2012 e 2013-2016, e o mandato do Prefeito Marcelo Crivella (2017-2020).

A uso da metodologia do estudo de caso permitiu investigação em profundidade da situação empiricamente singular da cidade do Rio de Janeiro por meio de múltiplas variáveis de interesse. A metodologia do estudo oferece a possibilidade de uso de fontes de informação diversificadas, como documentos públicos, dados de acesso aberto do Datasus e revisão de publicações científicas (livros, dissertações, teses e artigos científicos). A revisão bibliográfica possibilitou situar o contexto da implantação da terceirização por meio do modelo das OSS no município.

Para a demonstração do nível de prioridade na função saúde nas gestões da cidade, o artigo utiliza dados do Sistema de Informações sobre Orçamento Público em Saúde (SIOPS). Tomando como referência as indicações metodológicas de Pereira et al. ${ }^{12}$, o nível da vinculação financeira com ASPS é descrito a partir de duas equações:

A primeira equação permite calcular a despesa anual potencial em saúde per capita da cidade (DPSPC) com base nos parâmetros definidos pela Emenda Constitucional 29 de 2000, ou seja, esperava-se que o Município vinculasse no mínimo $15 \%$ das receitas próprias com saúde. O indicador da DPSPC deriva assim da equação: $(0,15$ X RPPC $)+(0,15$ X TCPC $)+$ TSUSPC, onde RPPC é a receita própria per capita; TCPC é a transferências constitucionais per capita e TSUSPC: é a transferência SUS per capita. Cabe lembrar que a Constituição Federal brasileira de 1988 (CF/88) instituiu mecanismos abrangentes de redistribuição das receitas tributárias e de contribuições entre os entes federativos ${ }^{13}$.

A segunda equação demonstra o nível de comprometimento da despesa do per capita com ASPS anualmente (NCDS). O NCDS deriva da razão DRPC/DPSPC, onde DPSPC é a despesa potencial em saúde per capita e DRPC é a despesa realizada per capita. O valor do NCDS igual ou $>1$, indica que a função saúde ocupa o topo da agenda de prioridade do governo. 
O dado sobre a mudança na estrutura do setor saúde por força da implantação do modelo das OSS foram extraídas do Plano de Saúde Municipal - 2013-2017

A descrição da expansão da ESF e dos seus desdobramentos nos indicadores de saúde nos três diferentes momentos do governo municipal baseou-se nas informações do Ministério da Saúde disponíveis no sítio e-SUS ${ }^{15}$. Para estimativa de pessoas contempladas pela equipe de saúde da família considerou-se que cada Equipe de Saúde da Família (ESF) era responsável em média por 3.450 pessoas (valor de referência utilizado à época pelo DAB/SAS/Ministério da Saúde) para o período de 2009 na 2019 conforme Programa Previne Brasil ${ }^{14}$.

\section{Resultados}

O modelo organizacional das OSS deriva da agenda da reforma do Estado de 1995 promovida pelo Ministro Bresser Pereira na década de $1990^{16}$. A agenda da reforma proposta definiu quatro núcleos de atividade do Estado brasileiro: (i) núcleo estratégico, conformado pelos Poderes Executivo, Legislativo, Judiciário, Forças Armadas, a serem mantidos como propriedade estatal e forma de administração mista burocrática e gerencial; (ii) atividades exclusivas do Estado, relacionadas a controle, fiscalização, seguridade, com propriedade estatal e administração gerencial; (iii) atividades não exclusivas de Estado, representadas pelas Universidades, hospitais públicos, centros de pesquisa, museus, adotando formas de propriedade "pública não-estatal" e administração gerencial por conversão ao modelo das OSS; e, finalmente, (iv) atividades voltadas à produção para o mercado, como as empresas estatais, a serem privatizadas ${ }^{17}$.

$\mathrm{Na}$ área social, a proposta das OSS visou, entre outros objetivos, reduzir a expansão da força de trabalho promovida pela implantação do Regime Jurídico Único (RJU) criado pela $\mathrm{CF} / 88$ e regulamentado pela Lei $8112 / 1990^{18}$. O RJU criou a figura do servidor público estatutário, diferenciando-o dos servidores públicos com contratos de trabalho pela Consolidação das Leis do Trabalho (CLT) e outros formatos abertamente precarizados.

Como mostra Marenco ${ }^{17}$, desde a $\mathrm{CF} / 88$ a força de trabalho da administração pública no Brasil pode ser classificada em quatro categorias excludentes: os estatutários, funcionários públicos contratados sob as regras do RJU, implicando seleção por meio de concurso público e a obtenção da estabilidade no cargo após três anos de estágio probatório; os celetistas, servidores com contrato de trabalho regido pela CLT, portadores de direitos trabalhistas e carteira de trabalho assinada, mas sem estabilidade na função pública; os funcionários comissionados para o exercício de funções de confiança política, de livre-nomeação pela autoridade governamental, podendo, igualmente, ser removidos por decisão desta autoridade; e os servidores "sem vínculo permanente" que trabalham por prestação de serviços, sem vínculo empregatício e sem carteira de trabalho assinada.

Por meio das OSS, a administração pública brasileira dispôs de mais uma opção organizacional para a expansão da força de trabalho no setor social sem a exigência abertura de concurso público e obediência ao teto dos salários das carreiras subordinadas ao RJU.

O novo modelo das OSS teve grande aceitação nos governos municipais e estaduais ${ }^{19-21}$, especialmente na área hospitalar ${ }^{22}$. Importante ressaltar que os níveis estaduais e municipais têm autonomia legislativa e puderam instituir as suas versões do novo modelo organizacional ${ }^{22}$.

Assim, as OSS foram muito atraentes para os gestores porque permitiram estabelecer a prestação serviços não exclusivos de Estado com entes privados por meio dos contratos de gestão ${ }^{23-25}$. Nos setores não exclusivos de Estado, a agenda da Reforma esperava que o contrato de gestão com terceiros ampliasse a autonomia, celeridade decisória e responsabilização, no sentido financeiro e organizacional.

É importante ressaltar que o fato da organização conseguir a qualificação como OSS, não necessariamente faz com que ela adquira o direito de celebrar o contrato de gestão com os governos. Antes disso, a OSS deve participar de um processo seletivo. Cada OSS deve apresentar uma proposta detalhada de trabalho, com orçamento e comprovação de experiência na área em que pretende atuar, entre outros requisitos explicitados em Edital público ${ }^{24}$.

Chama a atenção na experiência da cidade é que a expansão da atenção primária foi promovida por meio da adesão a agenda da reforma do Estado de 1995, na perspectiva da inovação organizacional, com a criação de condições de governabilidade para a nova agenda de gestão. Com efeito, um novo arcabouço jurídico foi aprovado pela Câmara de Vereadores da Cidade do Rio de Janeiro, a Lei Municipal no 5.026/2009. A adoção das OSS foi, assim, crucial para a viabilidade da agenda expansiva da APS na cidade, principal- 
mente por facultar a contratação dos profissionais das Equipes de Saúde da Família (ESF) pelo regime de CLT, reduzindo os vínculos trabalhistas precários existentes anteriormente nas relações do governo da cidade com a organizações não-governamentais ${ }^{25}$.

A Tabela 1 mostra que a aderência ao modelo das OSS foi acompanhada de um esforço significativo de vinculação das receitas próprias da cidade com a ASPS mensurado de indicador de comprometimento de receitas próprias. Em 2009, primeiro ano de governo o CDSPC foi 1,30 , indicando que o município dispendeu $30 \%$ acima da sua capacidade esperada de gasto com receitas próprias. Nos quatro anos seguintes (2010-2013), o CDPC permaneceu acima de 1, declinando residualmente nos dois últimos anos da gestão (20152016). Em 2017, primeiro ano da gestão Crivella, o CPC sofre uma redução monumental (foi de 0,88), mantendo-se abaixo de 1 nos dois anos de gestão seguintes (2018-2019). A Tabela 1 mostra que a despeito das despesas com saúde terem se mantido acima do parâmetro dos $15 \%$ esperados pela EC-29, o esforço fiscal da cidade com a função saúde foi significativamente reduzido nos três anos da gestão Crivella. Os dados para 2020 não estão disponíveis no SIOPS neste momento.

Os primeiros anos de implantação do modelo OSS no contexto de expansão da atenção primária tiveram também importante repercussão nas condições de provisão de atenção primária

Tabela 1. Nível de Comprometimento (NCDS) e Percentual de Despesas em Serviços Públicos de Saúde (ASPS) do Municípios do Rio de Janeiro segundo os parâmetros da Emenda Constitucional 29/2000, 20092019.

\begin{tabular}{ccc}
\hline Ano & $\begin{array}{c}\text { Nível de } \\
\text { Comprometimento } \\
\text { com Despesas ASPS }\end{array}$ & $\begin{array}{c}\text { \% de Aplicação da } \\
\text { EC29 }\end{array}$ \\
\hline 2009 & 1,30 & 16,07 \\
2010 & 1,01 & 16,91 \\
2011 & 1,05 & 19,69 \\
2012 & 1,01 & 23,25 \\
2013 & 1,0 & 19,43 \\
2014 & 0,98 & 20,81 \\
2015 & 0,95 & 20,93 \\
2016 & 0,93 & 25,48 \\
2017 & 0,88 & 25,71 \\
2018 & 0,94 & 21,1 \\
2019 & 0,99 & 20,64 \\
\hline
\end{tabular}

Fonte: SIOPS (https://www.saude.rj.gov.br/informacao-sus/ dados-sus/2019/01/indicadores). da cidade do Rio de Janeiro. Foi implantado um modelo híbrido neste nível de atenção, privilegiando as áreas extremamente pobres com vazio assistencial. A rede de atenção primária foi configurada por três tipos de unidades de saúde: do tipo A, onde todo o território é coberto por equipes da estratégia de Saúde da família); do tipo B (unidades de saúde tradicionais, com incorporação de uma ou mais equipes da ESF, que cobrem parcialmente o território); e tipo C (unidades básicas de saúde tradicionais, sem a presença de equipes de Saúde da família) ${ }^{6}$.

Os efeitos dessa rápida reorganização sobre a composição da força de trabalho foram superlativos em razão do expressivo crescimento dos vínculos celetistas nas OSS, vinculadas diretamente a implantação da ESF nos anos iniciais do primeiro mandato de Paes, como mostra a Tabela 2. Vale notar que a implantação das OSS foi acompanhada pela extinção da participação das cooperativas no desenvolvimento das intervenções de saúde da Prefeitura. No fim do primeiro mandato as OSS concentravam $31 \%$ do pessoal na prestação direta de atenção à saúde.

O Gráfico 1 demonstra que a evolução da cobertura da APS na cidade foi bastante consistente nos dois mandatos do Prefeito Paes (2009-2016). O conceito de APS no Gráfico 1 contempla os serviços da ESF somados à oferta das unidades básicas de saúde tradicionais da cidade. Vale assinalar que até 2009 o município do Rio de Janeiro organizava exclusivamente a APS em centros de saúde de formato tradicional (com médicos de especialidades básicas atendendo pessoas sem os instrumentos da adscrição, vínculo e acompanhamento). No primeiro ano do mandato do Prefeito Paes, iniciado em 2009, observou-se a célere implantação das clínicas de família dentro os parâmetros preconizados pela ESF. A Tabela 3 mostra a evolução quantitativa específica da cobertura e oferta de procedimentos de APS, que apresenta queda abrupta em 2019. A saída da saúde da agenda de governo na gestão Crivella deixou sem cobertura da ESF 880 mil pessoas entre 2016 e 2019. Entretanto, a Tabela 3 mostra também que a APS sofreu extraordinária queda da produtividade a partir de 2017. Em 2009, a produção de procedimentos de APS foi de 19,0 caindo para 2,5 por pessoa coberta pela ESF em 2019. A Tabela 3 mostra que o indicador permaneceu também acima de dois dígitos entre 20092016.

O período expansionista no gasto público, ampliação da cobertura e aumento da produtividade foi marcado sobretudo pela incorporação 
Tabela 2. Evolução da Força de Trabalho segundo os tipos de Vínculos na Secretaria Municipal de Saúde do Rio de Janeiro, 2009-2012.

\begin{tabular}{cccccccc}
\hline $\begin{array}{c}\text { Ano/ } \\
\text { Tipo de } \\
\text { vínculo }\end{array}$ & $\begin{array}{c}\text { Servidor } \\
\text { Estatutário } \\
\text { Municipal (A) }\end{array}$ & $\begin{array}{c}\text { Servidor } \\
\text { Estatutário } \\
\text { Federal (B) }\end{array}$ & $\begin{array}{c}\text { Cooperativas } \\
\text { com vínculos } \\
\text { precários }\end{array}$ & $\begin{array}{c}\text { Celetistas } \\
\text { em OSS }\end{array}$ & $\begin{array}{c}\text { Outros } \\
\text { (convênios } \\
\text { e FIOTEC) }\end{array}$ & Total & $\begin{array}{c}\% \\
\text { vinculados } \\
\text { às OSS }\end{array}$ \\
\hline 2009 & 25.411 & 5.338 & 932 & 0 & 2.963 & 34.644 & $0 \%$ \\
2010 & 25.048 & 5.218 & 0 & 5.015 & 2.040 & 37.321 & $13 \%$ \\
2011 & 24.422 & 5.072 & 0 & 8.549 & 2.692 & 40.735 & $21 \%$ \\
2012 & 24.504 & 4.673 & 0 & 14.427 & 3.222 & 46.856 & $31 \%$ \\
\hline
\end{tabular}

Fonte: Plano de Saúde Municipal, 2013-2017. Secretaria Municipal do Rio de Janeiro, 2013 (disponível em www.rio.rj.gov.br/web/ SMS).

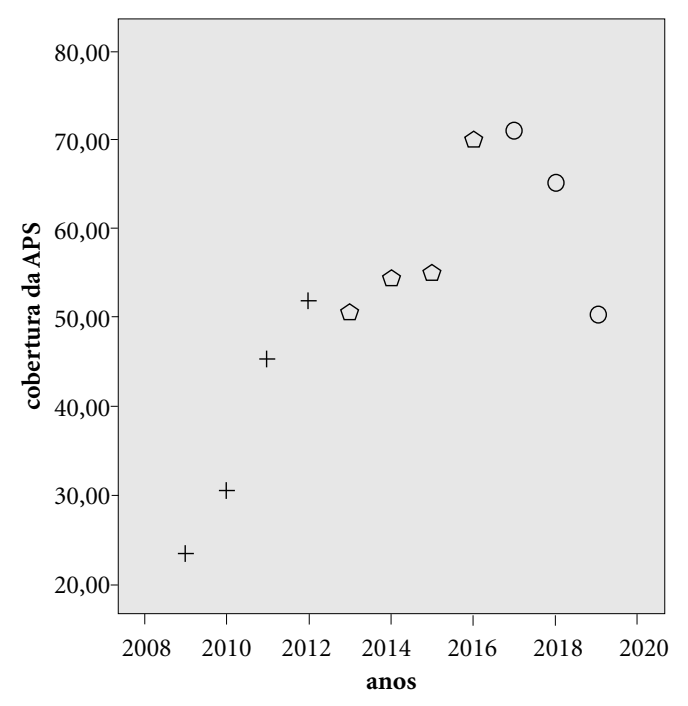

Governos

+ Primeiro Governo Paes

$\checkmark$ Segundo Governo Paes

Governo Crivella

Gráfico 1. Evolução da Cobertura da APS e os Mandatos dos Prefeitos Eduardo Paes e Marcelo Crivella na Cidade do Rio de Janeiro no Período 2009-2019.

Fonte: e-SUS (https://sisaps.saude.gov.br/painelsaps/saudefamilia, acessado em 18/10/2020).

da contratualização com o modelo organizacional da OSS, que trouxe para gestão municipal os incentivos para a avaliação e remuneração por desempenho e o objetivo da melhoria de qualidade, eficiência e transparência. O município também fortaleceu a área de medicina de família e comunidade (MFC), implantando a residência médica; equiparou a remuneração profissional aos padrões observados no mercado para as pro-
Tabela 3. Cobertura de ESF, Procedimentos de APS e Produção de atenção primária (APS) per capita, 20092019.

\begin{tabular}{cccc}
\hline Ano & $\begin{array}{c}\text { População } \\
\text { coberta } \\
\text { pela ESF } \\
\text { (A) }\end{array}$ & $\begin{array}{c}\text { Procedimentos } \\
\text { de APS (B) }\end{array}$ & $\begin{array}{c}\text { Procedimento } \\
\text { de APS per } \\
\text { capita (B/A) }\end{array}$ \\
\hline 2009 & 595.780 & 11.297 .786 & 19,0 \\
2010 & 990.820 & 12.975 .776 & 13,1 \\
2011 & 2.023 .099 & 20.670 .753 & 10,2 \\
2012 & 2.490 .935 & 26.667 .618 & 10,7 \\
2013 & 2.382 .286 & 28.446 .965 & 11,9 \\
2014 & 2.798 .317 & 33.122 .678 & 11,8 \\
2015 & 2.853 .604 & 39.175 .770 & 13,7 \\
2016 & 2.864 .898 & 37.185 .828 & 13,0 \\
2017 & 4.086 .903 & 36.820 .395 & 9,0 \\
2018 & 3.806 .849 & 17.258 .657 & 4,5 \\
2019 & 2.974 .458 & 7.555 .742 & 2,5 \\
\hline
\end{tabular}

Fonte: e-SUS (https://sisaps.saude.gov.br/painelsaps/saudefamilia, acessado em:18/10/2020).

fissões de saúde, modificou a regulação do acesso e uso de tecnologias de informação e comunicação ${ }^{26}$.

Cabe chamar a atenção que no primeiro período da gestão Paes (2009 a 2012) teve início a gestão das unidades de Atenção Primária à Saúde (APS) com o modelo de gestão via OSS ${ }^{27}$. O segundo período, de 2012 até 2016, a gestão pública viabilizou a expansão da escala de cobertura APS, chegando a 70\% da população em dezembro de 2016, segundo dados do Ministério da Saúde.

O terceiro período analisado é compreendido de 2017 a 2019, com governo de Marcelo Crivella que teve início sem a defesa do veto ao modelo das duas gestões anteriores, porém promoveu a 
redução da força de trabalho e o retraimento da cobertura da ESF, chegando a 50,5\% de cobertura de APS em dezembro de 2019. A gestão Crivella também optou pela extinção do modelo organizacional da via OSS e pela transferência da força de trabalho terceirizada na ESF para a Empresa Pública de Saúde - Rio Saúde (Fundação Estatal de Direito Privado). Note-se que mesmo com o veto ao modelo da OSS pela gestão Crivella, não foi feita a opção para de uma contrarreforma pela chamada de concursos públicos para a implantação da governança do RJU em larga escala na ESF da cidade.

\section{Discussão}

Neste artigo demonstrou-se que a sustentabilidade da adoção do modelo da OSS na cidade do Rio de Janeiro não dependeu da condição financeira da cidade, mas sim da prioridade que a função saúde teve na agenda política do governo municipal. O que singulariza a experiência da cidade do Rio de Janeiro é a notável expansão da AP por meio do modelo das OSS. Os ganhos de bem -estar social para a população pobre da cidade dessa experiência de ampliação da cobertura foram ignorados pela literatura, majoritariamente centrada na função de veto à escolha do governo municipal.

Nesse sentido, o estudo de caso da cidade do Rio de Janeiro demonstra também que, mesmo as experiências bem-sucedidas de expansão da APS com elevado comprometimento fiscal da cidade, podem sofrer severo retrocesso também em razão de veto de uma nova coalizão política à frente da gestão da cidade.

De fato, a experiencia do setor saúde nos dois primeiros mandatos de Eduardo Paes pode ser associado à alta priorização da função saúde no orçamento público, que destinou 25,5\% em 2016 dos recursos próprios no setor. Essa centralidade da saúde na agenda do governo foi acompanhada da definição da APS como ordenadora do sistema local de saúde, o que possibilitou ao SUS municipal alcançar 3,8 milhões de habitantes por meio da ESF em 2016 e obter elevados ganhos de eficiência alocativa.

As duas gestões de Eduardo Paes promoveram a reforma do setor e a expansão das equipes de saúde da família por meio da formalização de contratos de gestão com OSS habilitadas para gestão na saúde. Entretanto, o veto ${ }^{28}$ político ao arranjo da terceirização promovido pela gestão Crivella a partir de 2017 pode ser associada à redução da cobertura da ESF em quase 1 milhão de pessoas e à queda na produtividade setorial.

Os indicadores de cobertura demonstram que o veto ao modelo das OSS, substituído pela terceirização via empresa pública municipal, afetou as condições de acesso da população aos cuidados de saúde. O veto ao modelo deu-se a despeito da constatação de que as OSS favoreceram relações trabalhistas mais formalizadas (predominaram os vínculos celetistas) e os desenvolvimento de mecanismos de avaliação de desempenho das OSS, com vista a mitigar as trocas clientelistas e a corrupção na gestão local.

É importante registrar que a adoção do modelo OSS foi uma escolha bem-sucedida para a rápida implantação da sua agenda de governo para o SUS na cidade do Rio de Janeiro, ainda que não seja suficiente para desempenhar todas as funções essenciais da saúde pública. Suas limitações apontam para a necessidade de implementação de arranjos regulatórios independentes que reduzam a insegurança jurídica, o déficit informacional do Executivo e, principalmente, torne transparente para a sociedade civil e opinião pública as relações do setor público com terceiros.

\section{Colaboradores}

NR Costa, IM Silva, PT Lima, TS Silva, ICM Costa e IVO Figueiredo participaram igualmente de todas as etapas da elaboração do artigo. 


\section{Referências}

1. Faveret Filho P, Oliveira PJ. A universalização excludente: reflexões sobre as tendências do sistema de saúde. Planej Polit Publicas 1990; 3:139-162.

2. Soranz D, Pinto LP, Penna GO. Eixos e a Reforma dos Cuidados em Atenção Primária em Saúde (RCAPS) na cidade do Rio de Janeiro, Brasil. Cien Saude Colet 2016; 21(5):1327-1338.

3. Soranz D, Pisco LAC. Reforma dos Cuidados Primários em Saúde na cidade de Lisboa e Rio de Janeiro: contexto, estratégias, resultados, aprendizagem, desafios. Cien Saude Colet 2017; 22(3):679-686.

4. Costa NR, Pinto LF. Avaliação de programa de atenção à saúde: incentivo à oferta de atenção ambulatorial e a experiência da descentralização no Brasil. Cien Saude Colet 2002; 7(4):907-923.

5. Costa NR. A Estratégia de Saúde da Família, a atenção primária e o desafio das metrópoles brasileiras. Cien Saude Colet 2016; 21(5):1389-1398.

6. Secretaria Municipal de Saúde do Rio de Janeiro (SMS). Plano Municipal de Saúde [Internet]. 2013 [acessado 2020 dez 13]. Disponível em: www.rio.rj. gov.br/web/SMS.

7. Piola SF, França JRM, Nunes A. Os Efeitos da Emenda Constitucional 29 na alocação regional dos gastos públicos no Sistema Único de Saúde no Brasil. Cien Saude Colet 2016; 21(2):411-421.

8. Tsebelis G. Veto Players. How Political Institutions Work. Princeton: Princeton University Press; 2002.

9. Salles N, Guarnieri F. Estratégia eleitoral nos municípios brasileiros: componente programático e alinhamento partidário. Rev Sociol Polit 2019; 27(72):e001.

10. Portal G1. As Promessas de Crivella [Internet]. [acessado $2020 \mathrm{dez}$ 13]. Disponível em: http://especiais. g1.globo.com/rio-de-janeiro/2017/as-promessas-decrivella/\#/4-anos.

11. Prefeitura Municipal do Rio de Janeiro. Reorganização dos Serviços de APS - Estudo ara Otimização de Recursos. Rio de Janeiro: SMS-RJ/SUBPAV; 2018.

12. Pereira ATS, Campelo ACFS, Cunha FS, Noronha J, Cordeiro H, Dain S, Pereira TR. A sustentabilidade econômico-financeira no PROESF em municípios do Amapá, Maranhão, Pará e Tocantins. Cien Saude Colet 2006; 11(3):607-620.

13. Brasil. Constituição da República Federativa do Brasil de 1988. Diário Oficial da União 1988; 5 out

14. Brasil. Portaria no 2.979, de 12 de novembro de 2019. Institui o Programa Previne Brasil, que estabelece novo modelo de financiamento de custeio da Atenção Primária à Saúde no âmbito do Sistema Único de Saúde. Diário Oficial da União; 2019.

15. Brasil. Ministério da Saúde (MS). E-Gestor atenção básica. Informação e gestão da atenção básica [Internet]. 2020 [acessado 2020 out 20]. Disponível em: https://egestorab.saude.gov.br/paginas/acessoPublico/ relatorios/relHistoricoCoberturaAB.xhtml.

16. Bresser-Pereira LC. Reforma do Estado para a cidadania. São Paulo: Editora 34; 1998

17. Marenco A. Burocracias Profissionais Ampliam Capacidade Estatal para Implementar Políticas? Governos, Burocratas e Legislação em Municípios Brasileiros. Dados 2017; 60(4):1025-1058.
18. Modesto P, Cunha Junior LAP. Terceiro Setor e Parcerias na Área de Saúde. Belo Horizonte: Editora Fórum; 2011.

19. Bloise MS. Os gerentes de unidades básicas de saúde do município do Rio de Janeiro: perfil, vivências e desafios [dissertação]. Rio de Janeiro: ENSP/Fiocruz; 2015.

20. Brandão REA. A Implementação do Programa Saúde na Escola no território de Manguinhos (RJ): estratégias de privatização na política pública [dissertação]. Rio de Janeiro: EPSJV/ Fiocruz; 2014.

21. Flores PORA. Contratualização e a efetividade na assistência à saúde [dissertação]. Rio de Janeiro: FGV; 2016.

22. Silva VCE, Barbosa PR, Hortale VA. Parcerias na saúde: as Organizações Sociais como limites e possibilidades na gerência da Estratégia Saúde da Família. Cien Saude Colet 2016; 21(5):1365-1376.

23. Ramos ALP, Seta MHD. Atenção primária à saúde e Organizações Sociais nas capitais da Região Sudeste do Brasil: 2009 e 2014. Cad Saude Publica 2019; 35(4):e00089118.

24. Miranda CB. O problema de agência nos contratos de gestão: as organizações sociais de saúde do estado do Rio de Janeiro [dissertação]. Rio de Janeiro: FGV; 2015.

25. Carneiro CCG, Martins MIC. Novos modelos de gestão do trabalho no setor público de saúde e o trabalho do agente comunitário de saúde. Trab Educ Saude 2015; 13(1):45-66

26. Melo EA, Mendonça MHM, Teixeira M. A crise econômica e a atenção primária à saúde no SUS da cidade do Rio de Janeiro, Brasil. Cien Saude Colet 2019; 24(12):4593-4598.

27. Andreazzi MFS, Bravo MIS. Privatização da gestão e organizações sociais na atenção à saúde. Trab Educ Saude 2014; 12(3):499-518.

28. Sleman G. Crivella diz que OSs vão deixar de administrar unidades de saúde do Rio [Internet]. Band News; 2020 [acessado 2020 out 20]. Disponível em: https:// www.bandnewsfmrio.com.br/editorias-detalhes/crivella-diz-que-oss-vao-deixar-de-administra.

Artigo apresentado em 12/01/2021

Aprovado em 18/02/2021

Versão final apresentada em 20/02/2021

Editores-chefes: Maria Cecília de Souza Minayo, Romeu Gomes, Antônio Augusto Moura da Silva 